\title{
How is the VS (vessel plus surface) classification system applicable to magnifying narrow-band imaging examinations of gastric neoplasias initially diagnosed as low-grade adenomas?
}

\author{
Kenshi Yao
}

Published online: 13 March 2012

(c) The International Gastric Cancer Association and The Japanese Gastric Cancer Association 2012

The endoscopic diagnosis of gastric mucosal lesions comprises two steps: detection and then characterization. After an endoscopic diagnosis has been made, endoscopists insist on taking at least one forceps biopsy from the target lesion to allow histological confirmation, because the diagnostic accuracy of endoscopy using conventional white-light imaging (C-WLI) alone is not sufficient [1].

As so rightly pointed out in this month's issue of Gastric Cancer [1], we frequently encounter inconsistencies between the histological findings from biopsy specimens and endoscopically resected specimens. For example, the biopsy-based histological findings are of low-grade adenoma (LGA), but the resection-based histological findings are of high-grade adenoma (HGA) or early cancer (EC). This is because it is sometimes difficult for the pathologist to achieve a correct diagnosis from a small biopsy specimen alone, and it is impossible for the endoscopist to consistently obtain a target biopsy from the most suspicious part of the lesion referring to C-WLI endoscopy alone. Differentiating between LGA and HGA/EC is therefore difficult, even after histological biopsy-based examination. This can result in the overtreatment of lesions which are in fact LGAs. Dr. Kazuhiro Miwa's clinical question is pertinent. Guidelines for the endoscopic treatment of LGA on biopsy have not been established, and a solution to this problem is clinically relevant.

The development of narrow-band imaging (NBI) has enabled endoscopic optical biopsies throughout the gastrointestinal tract $[2,3]$. However, NBI is not suited to

K. Yao $(\bowtie)$

Department of Endoscopy, Fukuoka University Chikushi

Hospital, 1-1-1 Zokumyoin, Chikushino,

Fukuoka 818-8502, Japan

e-mail: yao@fukuoka-u.ac.jp lesion detection within the stomach because it is too dark to obtain an overview of the gastric mucosa due to the wide lumen of the stomach. On the other hand, magnifying narrow-band imaging (M-NBI) is a powerful tool for characterizing the gastric mucosal surface, because it can visualize the precise microanatomies of both the microvascular (MV) and microsurface (MS) patterns of gastric mucosal lesions (Table 1) [4]. Based on these visualized microanatomies, a VS (vessel plus surface) classification system has been proposed for differentiating between cancerous and noncancerous lesions [4].

The diagnostic system for ME was initially based upon the MV pattern alone [5]. In brief, the magnified endoscopic findings of early gastric cancer are (1) the presence of a demarcation line between a lesion and the background mucosa, and (2) the presence of an irregular MV pattern within the lesion [6]. Setting the criteria for cancer as the presence of both ME findings, we previously reported that $\mathrm{ME}$ is useful for differentiating between cancerous and noncancerous lesions that are small, flat, or depressed [7]. After incorporating NBI into ME, based on the MV pattern alone, we also reported previously that the sensitivity and the specificity of M-NBI were as high as 95.0 and $96.5 \%$, respectively, for small depressed mucosal lesions when we applied M-NBI after inspection under C-WLI [8].

Let us consider why determination of the MS pattern should be mandatory, even after we have demonstrated that the MV pattern alone is useful for characterizing flat or depressed gastric mucosal lesions by ME. The answer is that we sometimes encounter difficulties in visualizing the subepithelial MV architecture in cases of elevated-type epithelial neoplasia [9]. Accordingly, we established a comprehensive diagnostic system-the VS classification system-involving both the MV and MS patterns, in order to facilitate the diagnosis of neoplasias of all macroscopic 
types (depressed, flat, and elevated types). According to the VS classification system (Fig. 1), in cases where the MV pattern is not visualized, it is categorized as an absent MV pattern. In such cases, the MS pattern can be a very good alternative to the MV pattern. As shown in Fig. 9 of Dr. Kazuhiro Miwa's paper, the microvascular pattern may not be visualized at all, due to the presence of a white opaque substance (WOS) within the superficial part of the tumor that totally obscures the subepithelial microvascular pattern. To develop systematic diagnostic guidelines, we needed to analyze both the MV pattern and the MS pattern; i.e., the "VS classification."

Epithelial neoplasias of elevated type, identified as LGA by histology, often show this WOS [9]. When we only employed the MV pattern for our analysis, the diagnostic accuracy was lower than in cases where we employed the VS classification system. In fact, Tables 5 and 6 show that, with regard to elevated-type tumors, 5 out of $34 \mathrm{HGA} / \mathrm{EC}$

Table 1 Microanatomies of both the microvascular (MV) and microsurface (MS) patterns used in the VS classification system for gastric mucosal lesions

MV pattern
Capillary
Collecting venule (CV)
Microvessel (MV)
MS pattern
Marginal crypt epithelium (MCE)
Crypt-opening (CO)
Intervening part (IP)
White opaque substance (WOS)
Light blue crest (LBC)

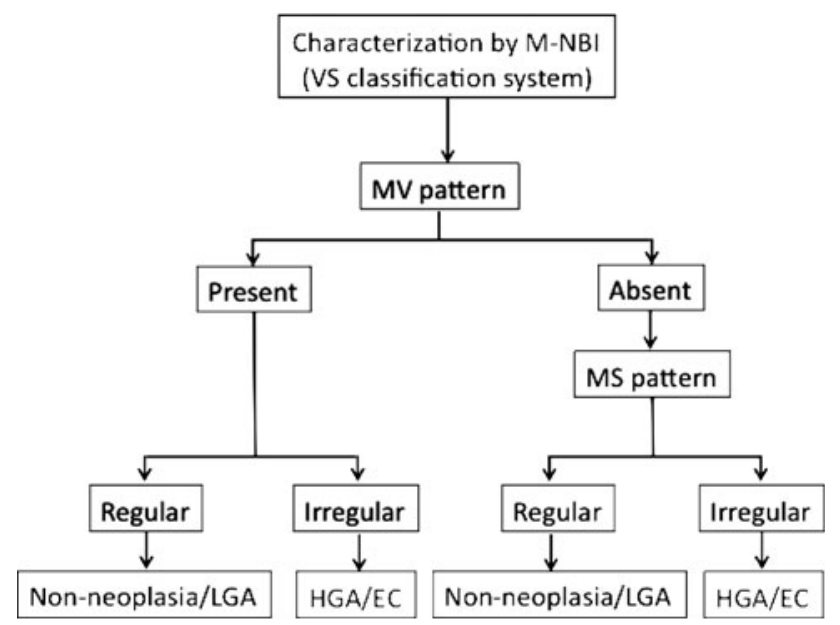

Fig. 1 How to characterize gastric mucosal lesions using M-NBI according to the VS classification system. M-NBI magnifying endoscopy, $M V$ microvascular, $M S$ microsurface, $H G A$ high-grade adenoma, $E C$ early cancer, $L G A$ low-grade adenoma and 12 out of 75 LGA showed an absent MV pattern, whereas for depressed-type tumors, 2 out of $24 \mathrm{HGA} / \mathrm{EC}$ and 2 out of 4 LGA showed an absent MV pattern. Overall, $21(16 \%)$ out of 135 lesions examined showed an absent MV pattern due to the presence of WOS. We can therefore state that one reason for the good diagnostic accuracy obtained was the use of both the MV and MS patterns. Although this is a retrospective study, the results are clinically relevant because we focused on a practical clinical question, and we propose a new strategy for improving the accuracy of diagnosis of gastric epithelial neoplasias of both elevated and depressed type (Fig. 2). Prospective studies will be required to test the real-time diagnostic performance of $\mathrm{M}-\mathrm{NBI}$ for gastric mucosal lesions of all macroscopic types.

In summary, the VS classification employs anatomical terms (Table 1). First, we should determine whether there is a demarcation line between the lesion and the background mucosa. Analyses of the MV and MS patterns should be made independently. The MV pattern is categorized as a regular/irregular/absent MV pattern, while the MS pattern is categorized as a regular/irregular/absent MS pattern. The original criteria for this VS classification system for cancer are as follows:

1. Presence of an irregular MV pattern with a demarcation line, or

2. Presence of an irregular MS pattern with a demarcation line.

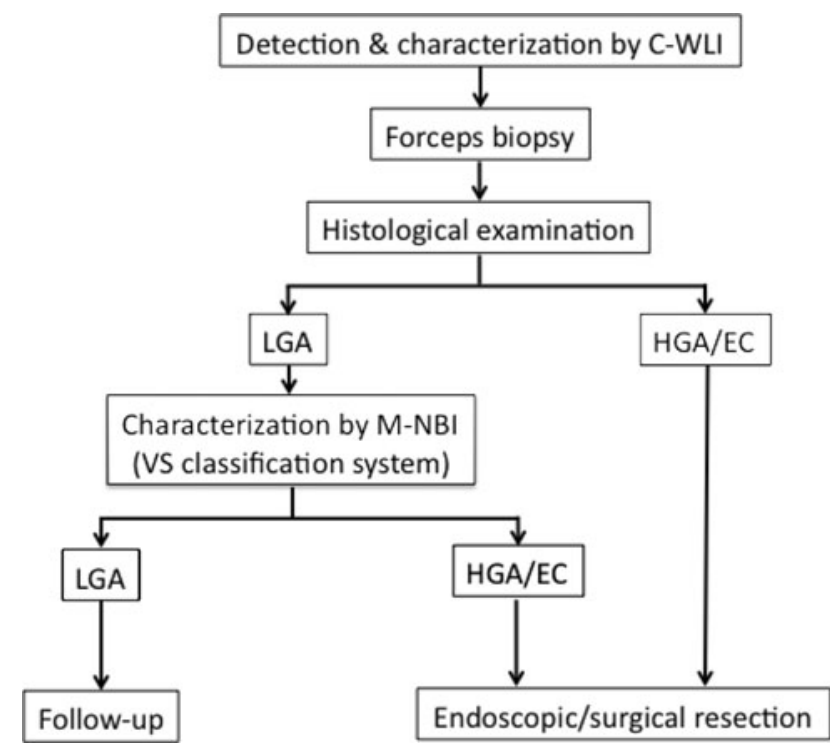

Fig. 2 Diagnostic and therapeutic strategies for gastric neoplastic lesions diagnosed as LGA by forceps biopsy with C-WLI. $C$-WLI conventional white light imaging, LGA low-grade adenoma, $H G A$ high-grade adenoma, $E C$ early cancer, $M-N B I$ magnifying narrowband imaging 
If (1) or (2) is present, the lesion is diagnosed as highgrade dysplasia/EC. Otherwise, the lesion is diagnosed as nonmalignant.

Since an irregular microvascular pattern is the most reliable marker of $\mathrm{HGA} / \mathrm{EC}$, we can propose a simple and efficient diagnostic algorithm (as shown in Fig. 1), based on numerous studies, including this article [1, 7-9]. In conclusion, this comprehensive VS classification system can be applied in order to establish guidelines for a treatment strategy for early gastric epithelial neoplasia using M-NBI.

\section{References}

1. Miwa $\mathrm{K}$, Doyama $\mathrm{H}$, Ito $\mathrm{R}$ et al. Can magnifying endoscopy with narrow band imaging be useful for low grade adenomas in preoperative biopsy specimens? Gastric Cancer. 2011. doi: 10.1007/s10120-011-0093-6

2. Yao K, Takaki Y, Matsui $\mathrm{T}$ et al. Clinical application of magnification endoscopy and narrow-band imaging in the upper gastrointestinal tract: new imaging techniques for detecting and characterizing gastrointestinal neoplasia. Gastrointest Endosc Clin N Am. 2008;18:415-433, vii-viii.

3. Katagiri $\mathrm{A}, \mathrm{Fu} \mathrm{KI}$, Sano $\mathrm{Y}$, et al. Narrow band imaging with magnifying colonoscopy as diagnostic tool for predicting histology of early colorectal neoplasia. Aliment Pharmacol Ther. 2008;27:1269-74.

4. Yao K, Anagnostopoulos GK, Ragunath K. Magnifying endoscopy for diagnosing and delineating early gastric cancer. Endoscopy. 2009;41:462-7.

5. Yao K, Iwashita A, Yao T. Early gastric cancer: proposal for a new diagnostic system based on microvascular architecture as visualized by magnified endoscopy. Dig Endosc. 2004;16:S110-7.

6. Yao K, Oishi $\mathrm{T}$, Matsui $\mathrm{T}$, Yao $\mathrm{T}$, et al. Novel magnified endoscopic findings of microvascular architecture in intramucosal gastric cancer. Gastrointest Endosc. 2002;56:279-84.

7. Yao K, Iwashita A, Tanabe H, et al. Novel zoom endoscopy technique for diagnosis of small flat gastric cancer: a prospective, blind study. Clin Gastroenterol Hepatol. 2007;5:869-78.

8. Ezoe Y, Muto M, Uedo N, et al. Magnifying narrowband imaging is more accurate than conventional white-light imaging in diagnosis of gastric mucosal cancer. Gastroenterology. 2011;141: 2017-25. doi:10.1053/j.gastro.2011.08.007.

9. Yao K, Iwashita A, Tanabe $\mathrm{H}$, et al. White opaque substance within superficial elevated gastric neoplasia as visualized by magnification endoscopy with narrow-band imaging: a new optical sign for differentiating between adenoma and carcinoma. Gastrointest Endosc. 2008;68:574-80. 